INPLASY

PROTOCOL

To cite: Zhang et al. Medial

Patellofemoral Ligament Reconstruction: A protocol for systematic review and meta analysis. Inplasy protocol 202180024. doi:

10.37766/inplasy2021.8.0024

Received: 07 August 2021

Published: 08 August 2021

Corresponding author:

Yong-qiang Zhang

1083367014@qq.com

Author Affiliation:

Shaanxi University of

Traditional Chinese Medicine.

Support: No.

Review Stage at time of this submission: Data analysis.

Conflicts of interest:

None declared.

\section{Medial Patellofemoral Ligament Reconstruction: A protocol for systematic review and meta analysis}

Zhang, YQ1; Li, P2; Zhang, Z3; Wu, M4; Zhou, YD5; Tao, SL6; Yang, YL7; Li, Y8; Liu, JL9; Teng, YS10; Guo, YM11.

Review question / Objective: Reconstruction of the medial patellofemoral ligament (MPFL) is an effective surgical method for the treatment of lateral patellar instability. At present, there is not much controversies regarding the femoral attachment, however, the controversies regarding patellar attachment versus attachment, number of graft strands, tension, isometry and so on. The following electronic databases will be searched: PubMed, the Cochrane Library, Embase, Web of Science, Medline. We will consider articles published between database initiation and March 2021. MPFL in the subject heading will be included in the study. Language is limited to English. Research selection, data extraction, and research quality assessment were independently completed by 2 researchers. By reviewing a large number of literature, this article summarizes the above problems during surgery and serves as a guideline to the management of MPFL reconstruction in lateral patellar instability.

INPLASY registration number: This protocol was registered with the International Platform of Registered Systematic Review and Meta-Analysis Protocols (INPLASY) on 07 August 2021 and was last updated on 07 September 2021 (registration number INPLASY202180024).

\section{INTRODUCTION}

Review question / Objective: Reconstruction of the medial patellofemoral ligament (MPFL) is an effective surgical method for the treatment of lateral patellar instability. At present, there is not much controversies regarding the femoral attachment, however, the controversies regarding patellar 
attachment versus attachment, number of graft strands, tension, isometry and so on. The following electronic databases will be searched: PubMed, the Cochrane Library, Embase, Web of Science, Medline. We will consider articles published between database initiation and March 2021. MPFL in the subject heading will be included in the study. Language is limited to English. Research selection, data extraction, and research quality assessment were independently completed by 2 researchers. By reviewing a large number of literature, this article summarizes the above problems during surgery and serves as a guideline to the management of MPFL reconstruction in lateral patellar instability.

Condition being studied: Medial Patellofemoral Ligament Reconstruction.

\section{METHODS}

Participant or population: Not reported.

Intervention: reconstruction of the medial patellofemoral ligament.

Comparator: Not reported.

Study designs to be included: Non-RCTs, quasi-RCTs, uncontrolled trials, reviews, case-controlledstudies, animal trials, and laboratory studies will be excluded.

Information sources: PubMed, the Cochrane Library, Embase, Web of Science, Medline.

Main outcome(s): The anatomy and biomechanics of MPFL, the stop point of MPFL reconstruction, the isometric of MPFL reconstruction, the anatomical and non-anatomical reconstruction of MPFL, and the tension of MPFL during reconstruction.

Quality assessment / Risk of bias analysis: PRISMA tools.

Subgroup analysis: Not reported.

Sensitivity analysis: Not reported.
Language: English.

Country(ies) involved: China.

Keywords: MPFL; reconstruction; patellar dislocation; patellar instability; femoral attachment.

Dissemination plans: MPFL; reconstruction; patellar dislocation; patellar instability; femoral attachment.

Contributions of each author:

Author 1 - Yong-qiang Zhang.

Author 2 - Peng Li.

Author 3 - Zhao Zhang.

Author 4 - Meng Wu.

Author 5 - Ya-dong Zhou.

Author 6 - Sheng-lin Tao.

Author 7 - Ya-long Yang.

Author 8 - Yang Li.

Author 9 - Jun-liang Liu.

Author 10 - Yun-sheng Teng.

Author 11 - Yong-ming Guo. 